Review Article

\title{
Opposing Effects of Oxygen Regulation on Kallistatin Expression: Kallistatin as a Novel Mediator of Oxygen-Induced HIF-1-eNOS-NO Pathway
}

\author{
Julie Chao, Youming Guo, Pengfei Li, and Lee Chao \\ Department of Biochemistry and Molecular Biology, Medical University of South Carolina, Charleston, SC, USA \\ Correspondence should be addressed to Julie Chao; chaoj@musc.edu
}

Received 17 August 2017; Accepted 25 October 2017; Published 13 December 2017

Academic Editor: Saeid Golbidi

Copyright (C) 2017 Julie Chao et al. This is an open access article distributed under the Creative Commons Attribution License, which permits unrestricted use, distribution, and reproduction in any medium, provided the original work is properly cited.

\begin{abstract}
Oxidative stress has both detrimental and beneficial effects. Kallistatin, a key component of circulation, protects against vascular and organ injury. Serum kallistatin levels are reduced in patients and animal models with hypertension, diabetes, obesity, and cancer. Reduction of kallistatin levels is inversely associated with elevated thiobarbituric acid-reactive substance. Kallistatin therapy attenuates oxidative stress and increases endothelial nitric oxide synthase (eNOS) and NO levels in animal models. However, kallistatin administration increases reactive oxygen species formation in immune cells and bacterial killing activity in septic mice. High oxygen inhibits kallistatin expression via activating the JNK-FOXO1 pathway in endothelial cells. Conversely, mild oxygen/hyperoxia stimulates kallistatin, eNOS, and hypoxia-inducible factor-1 (HIF-1) expression in endothelial cells and in the kidney of normal mice. Likewise, kallistatin stimulates eNOS and HIF-1, and kallistatin antisense RNA abolishes oxygen-induced eNOS and HIF-1 expression, indicating a role of kallistatin in mediating mild oxygen's stimulation on antioxidant genes. Protein kinase C (PKC) activation mediates HIF-1-induced eNOS synthesis in response to hyperoxia/exercise; thus, mild oxygen through PKC activation stimulates kallistatin-mediated HIF-1 and eNOS synthesis. In summary, oxidative stress induces down- or upregulation of kallistatin expression, depending on oxygen concentration, and kallistatin plays a novel role in mediating oxygen/exercise-induced HIF-1-eNOSNO pathway.
\end{abstract}

\section{Introduction}

Kallistatin was first identified in human plasma as a tissue kallikrein-binding protein (KBP) and characterized as a serine proteinase inhibitor (serpin) [1-3]. Tissue kallikrein (TK) is a serine proteinase that cleaves low molecular weight kininogen substrate to release vasodilating kinin peptides [4]. Kallistatin consists of two structural elements, an active site and a heparin-binding domain, which exert pleiotropic activities by regulating differential signaling pathways $[5,6]$. Kallistatin through its active site forms a covalent complex with TK and inhibits TK activity [5,7]. Kallistatin via its heparin-binding site interacts with cell surface heparan sulfate proteoglycans and thereby antagonizes the signaling pathways mediated by vascular endothelial growth factor, tumor necrosis factor- $\alpha$, transforming growth factor- $\beta$, and Wnt [8-11]. Moreover, kallistatin exerts a wide spectrum of biological effects independent of TK. For example, kallistatin is a potent vasodilator unrelated to the tissue kallikrein-kinin system [12]. Transgenic mice overexpressing kallistatin have lower blood pressure compared to control mice and are resistant to lipopolysaccharideinduced mortality $[13,14]$. Kallistatin is mainly expressed in the liver and is widely distributed in the kidney, heart, and blood vessel [15-18]. Circulating kallistatin levels are markedly reduced under pathological conditions, such as in hypertension, liver disease, sepsis, cardiac and renal injury, severe pneumonia, obesity, and cancer in patients and in animal models [19]. Kallistatin administration by gene or protein delivery alleviates hypertension, multiorgan 


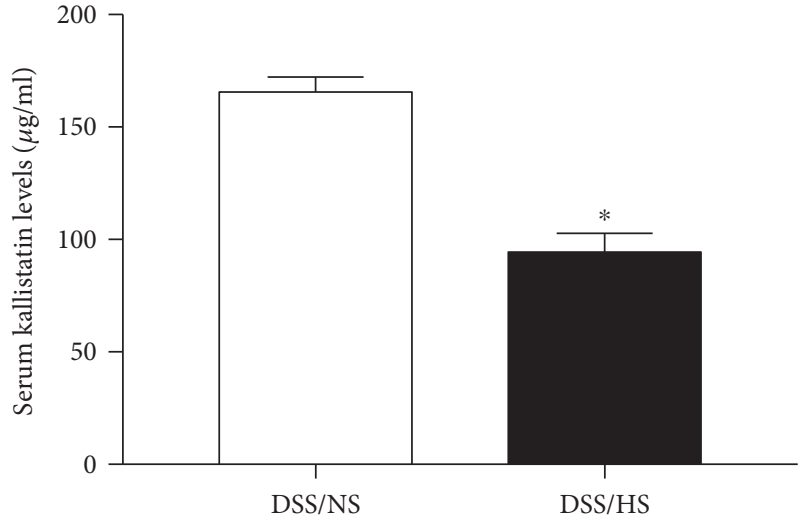

(a)

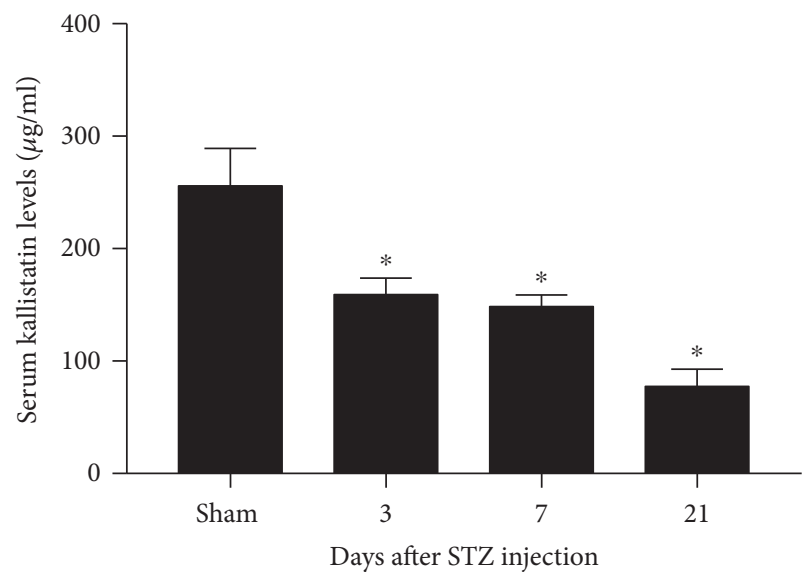

(c)

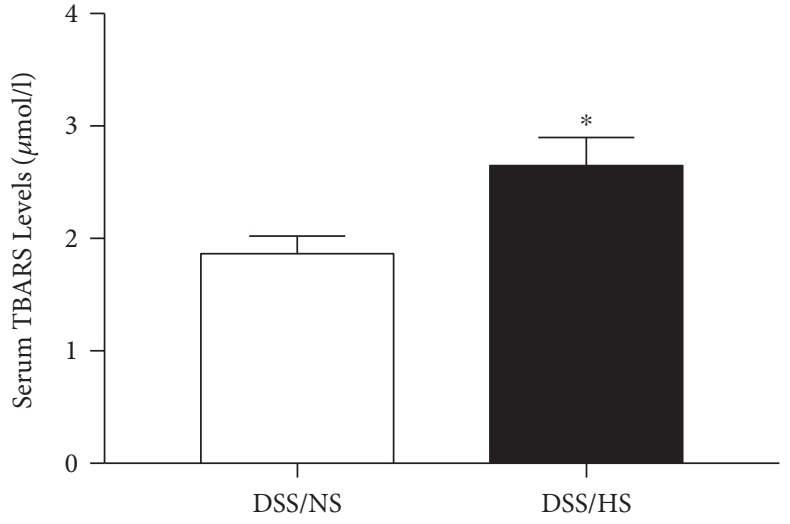

(b)

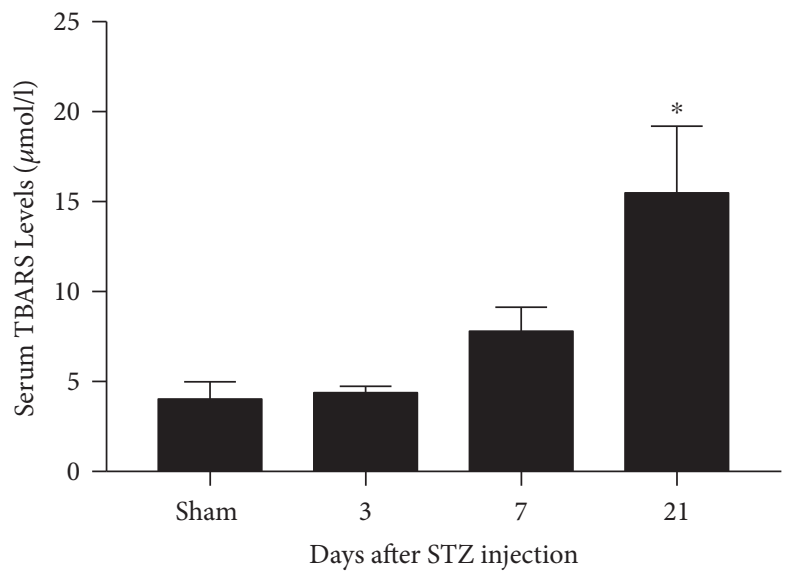

(d)

FIGURE 1: Circulating kallistatin levels are reduced, and TBARS levels are increased in DSS hypertensive rats and STZ-induced diabetic rats. ${ }^{*} P<0.05$ versus control group.

damage, and cancer development by reducing oxidative stress, inflammation, angiogenesis, apoptosis, fibrosis, tumor growth, and metastasis in rodents [8,20-25]. These findings indicate that kallistatin therapy has beneficial effects in various disease states.

Kallistatin belongs to the serpin family, which includes $\alpha 1$-antitrypsin and $\alpha 1$-antichymotrypsin [2]. In contrast to $\alpha 1$-antitrypsin, kallistatin is a negative acute-phase protein [26]. Kallistatin levels are markedly reduced in animals after endotoxin shock or experimental inflammation [26]. Oxidative stress downregulates kallistatin expression by activating c-Jun $\mathrm{NH}_{2}$-terminal kinase- (JNK-) dependent FOXO1 signaling in cultured endothelial cells [27]. However, hyperoxia treatment markedly stimulates kallistatin expression in breast cancer cells [28]. Moreover, kallistatin exhibits antioxidative actions. Kallistatin via its heparin-binding site antagonizes cytokine-induced reactive oxygen species (ROS) formation, and its active site is responsible for the upregulation of antioxidant gene expression in endothelial cells $[9,10$, 29]. On the other hand, kallistatin stimulates ROS formation in immune cells, leading to marked bacterial killing activity in septic mice $[28,30]$. Moreover, kallistatin's vasodilating activity is partly mediated by $\mathrm{H}_{2} \mathrm{O}_{2}$ formation [28]. Therefore, oxidative stress plays opposite roles in the regulation of kallistatin synthesis, and kallistatin possesses a dual role in modulating oxidative stress.

\section{Reduced Circulating Kallistatin Levels Are Inversely Associated with Oxidative Stress}

Circulatory kallistatin levels are markedly reduced in spontaneous hypertensive and arterial hypertensive rats $[15,31,32]$. Reduced kallistatin levels are associated with increased oxidative organ damage in animal models of hypertension and cardiovascular and renal dysfunction. Likewise, plasma kallistatin levels are reduced in patients with liver disease, sepsis, pulmonary pneumonia, obesity, and cancer [19]. Moreover, serum kallistatin levels are decreased in Dahl salt-sensitive (DSS) hypertensive rats receiving a high-salt diet (HS), compared with DSS rats with a normal-salt diet (NS), and are reduced time dependently in rats receiving streptozotocin (STZ) injection, a model of diabetes (Figures 1(a) and 1(c)). Reduced kallistatin levels are inversely associated with elevated serum thiobarbituric acid reactive substances (TBARs, an indicator of lipid peroxidation) in hypertensive DSS rats and in STZ-induced diabetic rats (Figures 1(a), 1(b), 1(c), and 1(d)). These findings indicate that 


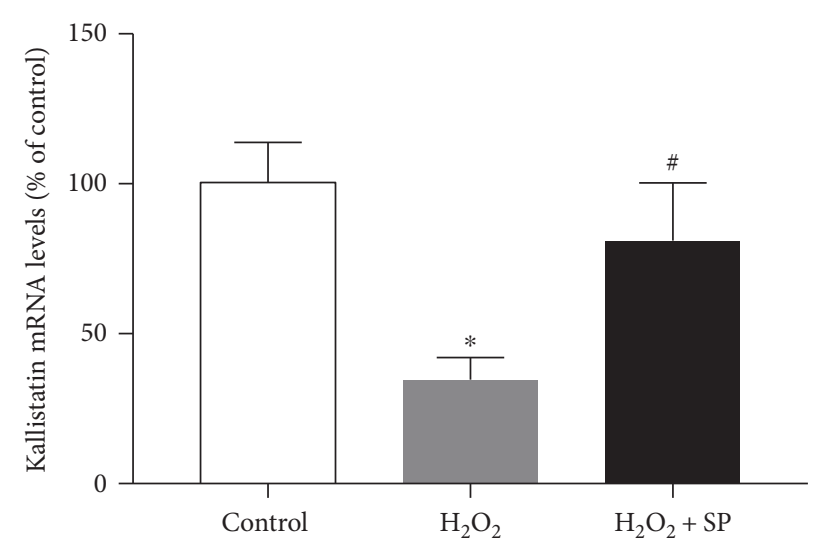

(a)

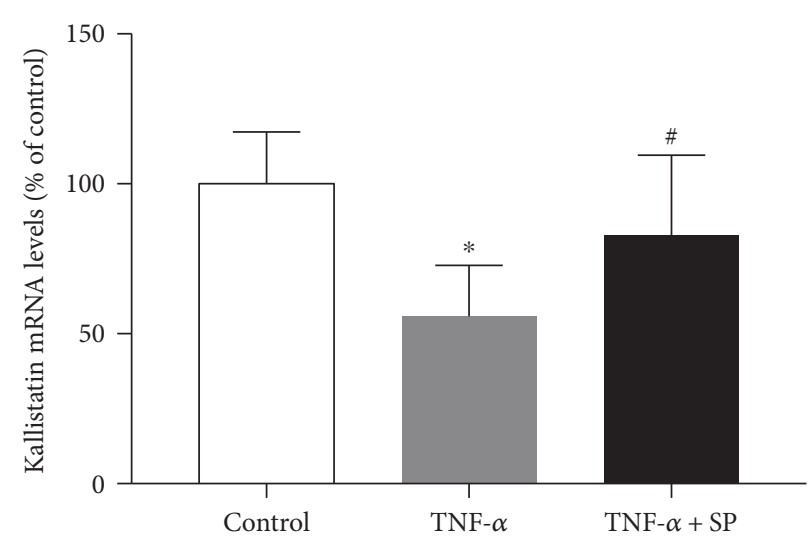

(b)

Figure 2: High $\mathrm{H}_{2} \mathrm{O}_{2}$ concentration $(100 \mu \mathrm{M}, 24 \mathrm{hr})$ or TNF- $\alpha(10 \mathrm{ng} / \mathrm{ml}, 24 \mathrm{hr})$ inhibits kallistatin expression in human umbilical vein endothelial cells (HUVECs), which is blocked by SP, a JNK inhibitor. ${ }^{*} P<0.05$ versus control group; ${ }^{\#} P<0.05$ versus $\mathrm{H}_{2} \mathrm{O}_{2}$ or TNF- $\alpha$ group.

kallistatin levels are reduced in diseased states and, consequently, negatively associated with oxidative stress.

\section{Kallistatin Treatment Reduces Oxidative Stress and Organ Damage}

Oxidative stress is a key contributor to the pathogenesis of hypertension, inflammation, fibrosis, and multiorgan injury [33-35]. Kallistatin administration attenuates cardiovascular and renal damage associated with reduced superoxide formation, inflammation, and increased endothelial nitric oxide synthase (eNOS) and NO levels in animal models of acute and chronic myocardial damage and salt-induced hypertension [21, 36, 37]. Moreover, kallistatin treatment inhibits liver fibrosis via antioxidative stress [38]. Conversely, depletion of endogenous kallistatin by neutralizing antibody injection augments cardiovascular and renal injury, in conjunction with increased oxidative stress, inflammation, endothelial cell loss, and fibrosis in hypertensive rats [39]. Kallistatin acts as a potent antioxidant as it prevents oxidative NO inactivation induced by superoxide production in cultured renal epithelial tubular and mesangial cells, cardiomyocytes, myofibroblasts, endothelial cells, and endothelial progenitor cells (EPCs) [21, 27, 29, 37, 40]. Moreover, kallistatin exhibits antioxidant activity in cultured pterygium epithelial cells through inhibition of ROS formation [41]. Kallistatin's heparin-binding site is crucial for blocking tumor necrosis factor- (TNF-) $\alpha$-induced NADPH oxidase activity and expression, and its active site is a key for stimulating the activity and expression of the antioxidant enzymes, eNOS, sirtuin 1 (SIRT1), and catalase in endothelial cells and EPCs $[9,10,29]$. Collectively, kallistatin protects against multiorgan damage through its antioxidative actions.

\section{High Oxygen Downregulates Kallistatin Expression}

Oxidative stress effectively suppresses kallistatin expression in vivo and in vitro. Kallistatin synthesis is rapidly diminished in the liver of rats after endotoxin shock [26]. In cultured endothelial cells, high $\mathrm{H}_{2} \mathrm{O}_{2}$ concentration at 100 or $200 \mu \mathrm{M}$ significantly reduces kallistatin mRNA and protein levels by reverse transcription polymerase chain reaction and Western blot analysis [27]. Likewise, high $\mathrm{H}_{2} \mathrm{O}_{2}$ concentration $(100 \mu \mathrm{M}, 24$ hours) or TNF- $\alpha$ $(10 \mathrm{ng} / \mathrm{ml}, 24$ hours) markedly inhibits kallistatin expression, and the inhibitory effect of high $\mathrm{H}_{2} \mathrm{O}_{2}$ or TNF- $\alpha$ on kallistatin expression is abolished by SP, a JNK inhibitor in endothelial cells (Figures 2(a) and 2(b)). Moreover, knockout of FOXO1 with antisense RNA has been shown to block oxidative stress-mediated suppression of kallistatin synthesis in endothelial cells [27]. Therefore, oxidative stress activates the JNK-dependent FOXO1 signaling pathway leading to the inhibition of kallistatin expression.

\section{Mild Oxygen or Hyperoxia Upregulates Kallistatin, HIF-1, and eNOS Expression}

The physiology oxygen levels differ under in vitro and in vivo conditions. High $\mathrm{H}_{2} \mathrm{O}_{2}$ concentration $(100 \mu \mathrm{M})$ inhibits kallistatin expression (Figure 2). In contrast to high $\mathrm{H}_{2} \mathrm{O}_{2}$, low or mild $\mathrm{H}_{2} \mathrm{O}_{2}$ concentrations (1 to $30 \mu \mathrm{M}$ for 12 hours) dose dependently stimulate kallistatin synthesis in endothelial cells (data not shown). The stimulatory effect of $\mathrm{H}_{2} \mathrm{O}_{2}$ $(10 \mu \mathrm{M})$ on kallistatin expression is associated with increased eNOS and HIF-1 synthesis (Figures 3(a), 3(b), and 3(c)). Likewise, hyperoxia ( $95 \% \mathrm{O}_{2} / 5 \% \mathrm{CO}_{2}$ for 6 hours) stimulates the expression of kallistatin, eNOS, and HIF-1 in endothelial cells (Figures 3(d), 3(e), and 3(f)). In the in vivo hyperoxia model, mice were placed in a $95 \% \mathrm{O}_{2} / 5 \% \mathrm{CO}_{2}$ chamber for 90 minutes as described [42]. Hyperoxia treatment increases kallistatin, eNOS, and HIF-1 mRNA levels in the mouse kidney (Figures 3(g), 3(h), and 3(i)). Hyperbaric oxygen therapy has been shown to prolong survival of mice with systemic metastatic cancer, reduces the growth, and induces apoptosis in rat mammary tumors [43]. Indeed, our previous study showed that hyperoxia markedly induces kallistatin expression in MDA-MB-231 and MCF-7 breast cancer cells and kallistatin treatment inhibits tumor progression by inducing cancer cell apoptosis and autophagy $[11,28]$. Therefore, mild 


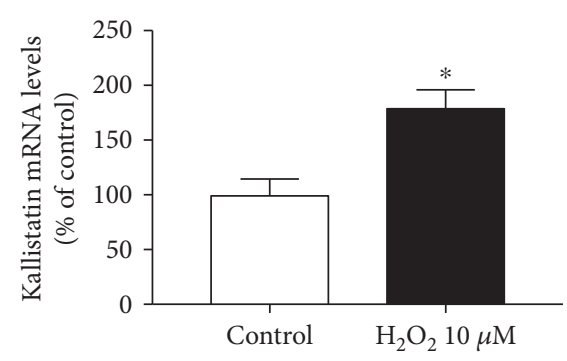

(a)

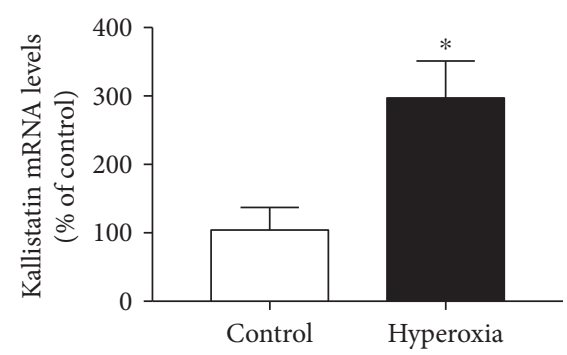

(d)

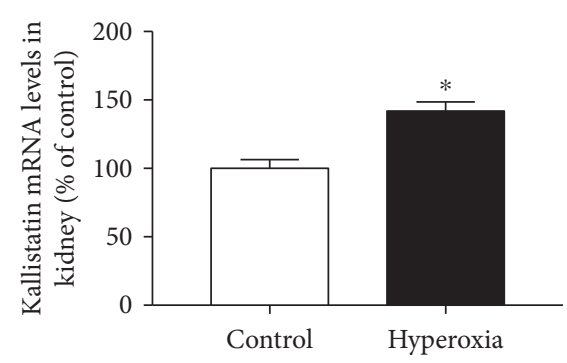

(g)

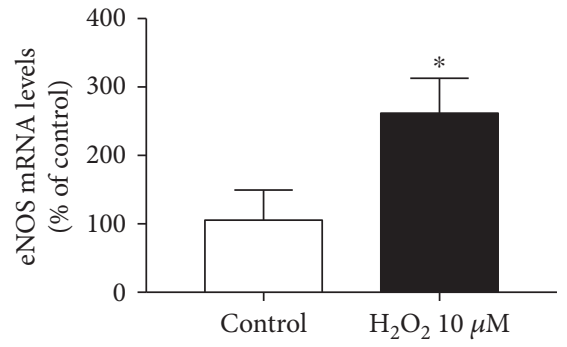

(b)

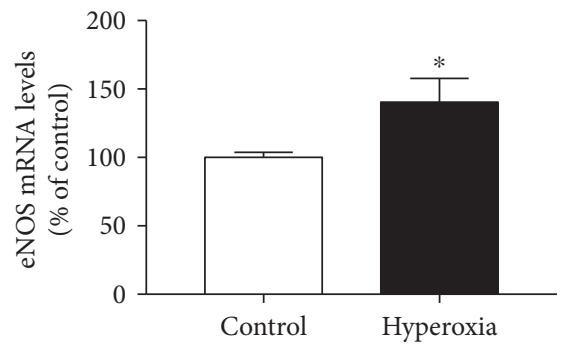

(e)

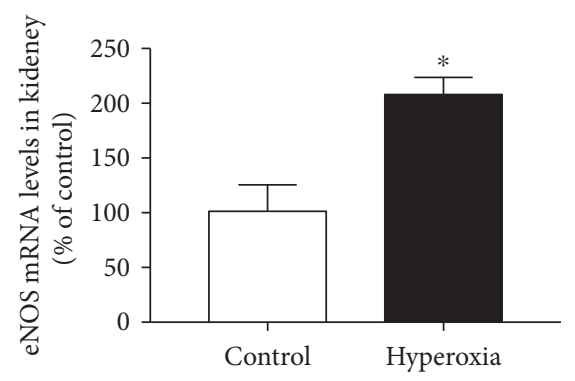

(h)

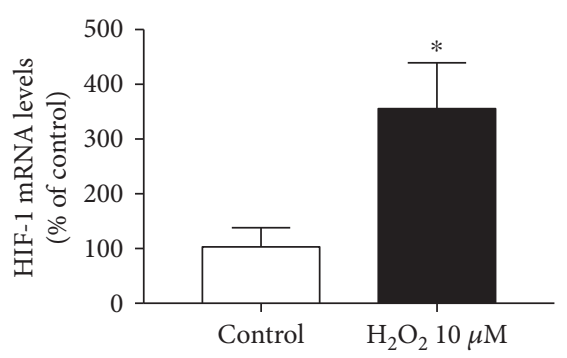

(c)

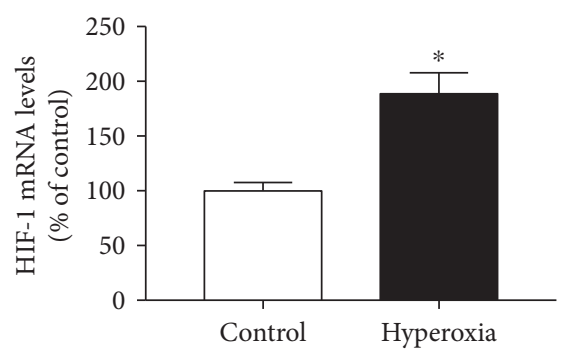

(f)

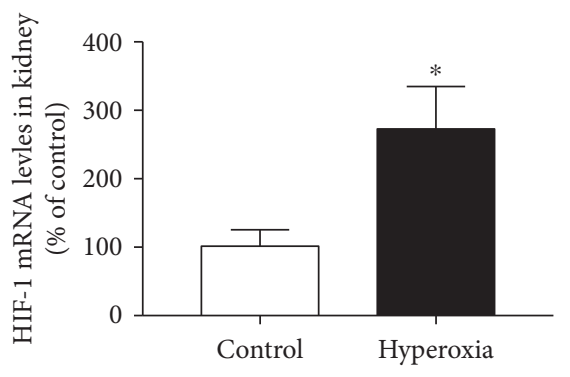

(i)

Figure 3: Mild $\mathrm{H}_{2} \mathrm{O}_{2}(10 \mu \mathrm{M}, 12 \mathrm{hr})$ or hyperoxia ( $95 \% \mathrm{O}_{2} / 5 \% \mathrm{CO}_{2}, 6 \mathrm{hr}$ for endothelial cells and 90 min for mice) stimulates kallistatin, eNOS, and HIF-1 synthesis in endothelial cells and in the kidney of mice. ${ }^{*} P<0.05$ versus control group.

oxygen or hyperbaric oxygen upregulates kallistatin, HIF-1, and eNOS expression and thus NO formation.

\section{Kallistatin Mediates Mild Oxygen-Induced HIF-1 and eNOS Synthesis}

In parallel with mild oxygen/hyperoxia, kallistatin is capable of stimulating eNOS and HIF-1 synthesis in endothelial cells (Figures 4(a) and 4(b)). Importantly, kallistatin not only elevates eNOS mRNA level but also increases eNOS activity and immunostaining, as well as NO formation in EPCs and endothelial cells $[25,40]$. Moreover, kallistatin antisense RNA abolishes $\mathrm{H}_{2} \mathrm{O}_{2}$-induced eNOS and HIF-1 synthesis, indicating a role of kallistatin in mediating mild oxygen-induced eNOS and HIF-1 synthesis (Figures 4(c), 4(d), and 4(e)). Mild hyperoxia via protein kinase C (PKC) activation increases HIF-1 synthesis, and eccentric exercise induces HIF-1-mediated-eNOS expression [44-48]. Taken together, these findings indicate that kallistatin is a novel mediator of mild oxygen-induced HIF-1 and eNOS synthesis, and mild oxygen through PKC activation stimulates kallistatinmediated HIF-1-eNOS pathway.

\section{Kallistatin Protects against Vascular and Organ Injury by Mediating Oxygen/Exercise- Induced HIF-1-eNOS-NO Pathway}

Physical activity and exercise training lower blood pressure in individuals with hypertension [49]. Moreover, exercise training exerts beneficial effects in diabetes and attenuates the decline of immune function associated with aging [50, 51]. Regular exercise prevents oxidative stress-related diseases, while acute exercise increases free-radical generation and oxidative injury in the elderly [52-54]. Older men who exercise on a regular basis do not demonstrate ageassociated vascular oxidative stress [54]. Indeed, exercise prevents aging-induced decline of eNOS/NO in the aorta [55], and regular physical activity improves endothelial function in patients with coronary artery disease by increasing eNOS phosphorylation and in animals with elevated NO levels [56]. Likewise, regular aerobic exercise restores endothelial function in arteries of aged mice by reducing oxidative stress, increasing superoxide dismutase activity, and downregulating NADPH oxidase activity [57]. Kallistatin treatment attenuates vascular senescence and aging by suppression of oxidative stress and stimulation of antioxidant gene 


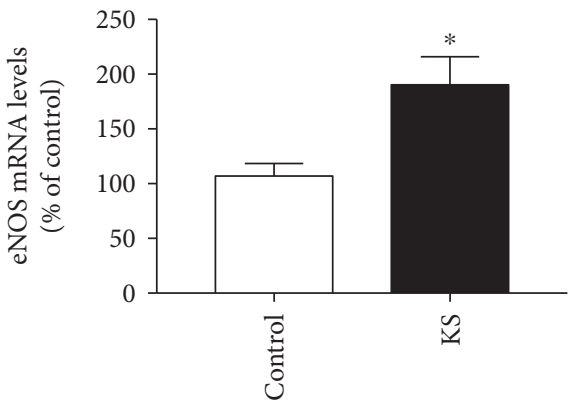

(a)

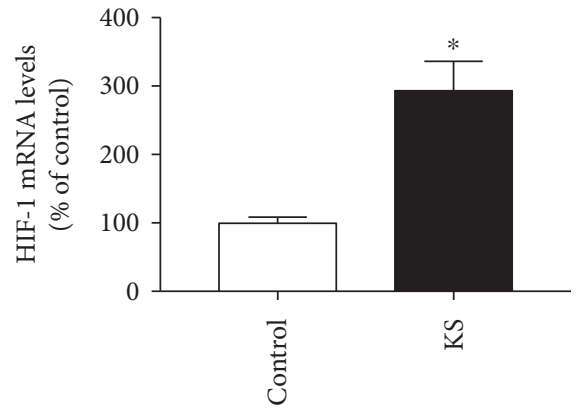

(b)

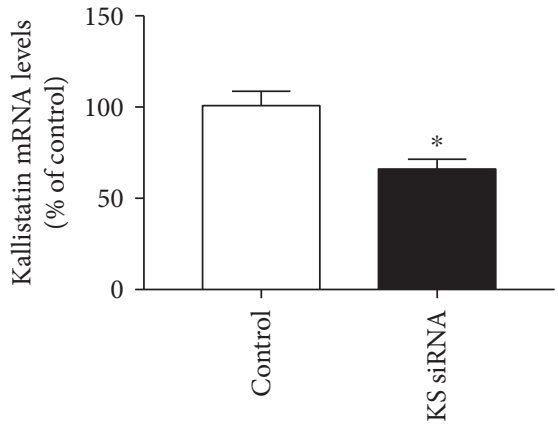

(c)

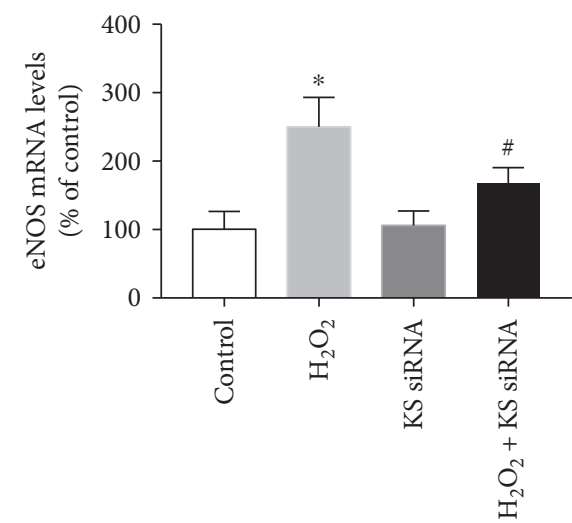

(d)

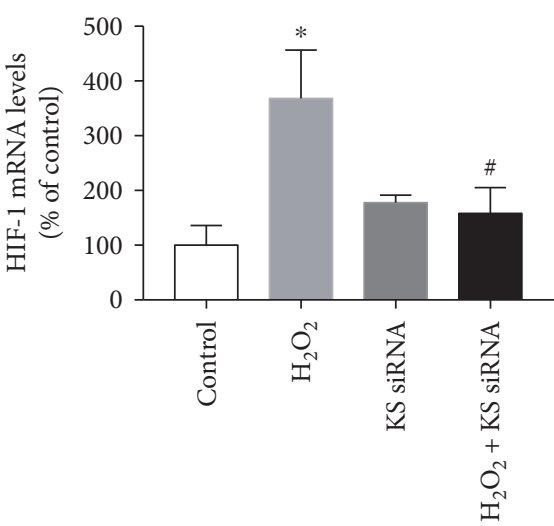

(e)

FIGURE 4: Kallistatin stimulates eNOS and HIF-1 expression and mediates mild oxygen-induced eNOS and HIF-1 synthesis in endothelial cells. ${ }^{*} P<0.05$ versus control group; ${ }^{\#} P<0.05$ versus $\mathrm{H}_{2} \mathrm{O}_{2}$ group.

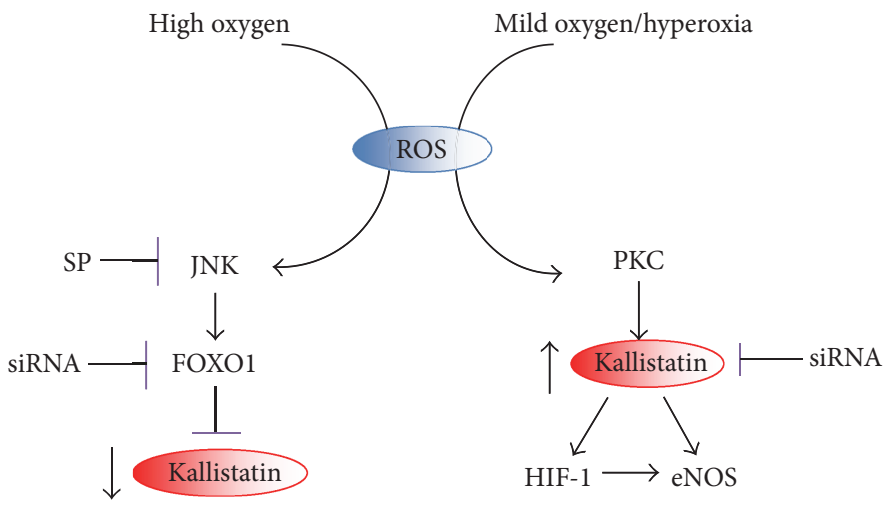

FIgURE 5: Signaling pathways mediated by high or mild oxygen on kallistatin regulation. High oxygen through ROS generation stimulates JNK/FOXO1 signaling activation, leading to kallistatin inhibition; mild oxygen/hyperoxia through PKC activation stimulates kallistatinmediated HIF-1 and eNOS synthesis in endothelial cells.

expression [29]. Moreover, kallistatin by stimulation of eNOS expression and activity and NO formation leads to blood pressure lowering, antioxidant and anti-inflammatory actions, and antiaging effect $[9,19,21,27,29]$. Therefore, regular exercise/mild oxygen increases kallistatin expression, leading to activation of HIF-1-eNOS-NO signaling. Collectively, kallistatin plays a protective role in vascular and organ injury by mediating oxygen/exercise-induced HIF-1-eNOSNO pathway.

\section{Conclusion}

Oxidative stress exerts opposite effects in regulating kallistatin expression. High $\mathrm{H}_{2} \mathrm{O}_{2}$ concentration $(100 \mu \mathrm{M})$ inhibits kallistatin expression via activating the JNK-dependent signaling pathway, while low or mild oxygen $\left(\mathrm{H}_{2} \mathrm{O}_{2}\right.$ at 1 to $30 \mu \mathrm{M}$ ) and mild hyperoxia via PKC activation stimulate kallistatin-mediated HIF-1 and eNOS synthesis (Figure 5). Moreover, kallistatin possesses both antioxidant and 
prooxidant actions. Reduced kallistatin levels in the circulation or tissues are inversely correlated with elevated superoxide levels, and kallistatin treatment attenuates oxidative stress and organ damage. In contrast, kallistatin stimulates ROS formation in immune cells and causes marked bacterial killing activity in septic mice. Kallistatin stimulates eNOS and HIF-1 synthesis, and depletion of kallistatin by kallistatin antisense RNA blocks mild oxygen-induced eNOS and HIF-1 synthesis. HIF-1 modulates eNOS expression induced by exercise training. Therefore, kallistatin acts as a novel mediator of oxygen/exercise-induced HIF-1eNOS-NO pathway and protects against oxidative vascular and organ injury. In summary, these findings reveal two important messages: (1) oxygen at high or low concentration exerts opposing effects on kallistatin expression and (2) kallistatin, by its dual role in regulation of oxidative stress, displays beneficial effects in pathological conditions such as hypertension, cardiovascular and renal injury, sepsis, and cancer development.

\section{Conflicts of Interest}

The authors declare that there is no conflict of interests regarding the publication of this paper.

\section{Authors' Contributions}

Julie Chao contributes to the writing of the paper, and Youming Guo, Pengfei Li, and Lee Chao contribute to the revision and citation of the paper.

\section{Acknowledgments}

This work was supported by the National Institutes of Health Grants HL118516 and HL44083.

\section{References}

[1] J. Chao, D. M. Tillman, M. Y. Wang, H. S. Margolius, and L. Chao, "Identification of a new tissue-kallikrein-binding protein," The Biochemical Journal, vol. 239, no. 2, pp. 325-331, 1986.

[2] J. Chao, K. X. Chai, L. M. Chen et al., "Tissue kallikreinbinding protein is a serpin. I. Purification, characterization, and distribution in normotensive and spontaneously hypertensive rats," The Journal of Biological Chemistry, vol. 265, no. 27, pp. 16394-16401, 1990.

[3] G. X. Zhou, L. Chao, and J. Chao, "Kallistatin: a novel human tissue kallikrein inhibitor. Purification, characterization, and reactive center sequence," The Journal of Biological Chemistry, vol. 267, no. 36, pp. 25873-25880, 1992.

[4] K. D. Bhoola, C. D. Figueroa, and K. Worthy, "Bioregulation of kinins: kallikreins, kininogens, and kininases," Pharmacological Reviews, vol. 44, no. 1, pp. 1-80, 1992.

[5] V. C. Chen, L. Chao, and J. Chao, "Reactive-site specificity of human kallistatin toward tissue kallikrein probed by sitedirected mutagenesis," Biochimica et Biophysica Acta (BBA) Protein Structure and Molecular Enzymology, vol. 1479, no. 1-2, pp. 237-246, 2000.
[6] V. C. Chen, L. Chao, D. C. Pimenta, G. Bledsoe, L. Juliano, and J. Chao, "Identification of a major heparin-binding site in kallistatin," The Journal of Biological Chemistry, vol. 276, no. 2, pp. 1276-1284, 2001.

[7] V. C. Chen, L. Chao, and J. Chao, "Roles of the P1, P2, and P3 residues in determining inhibitory specificity of kallistatin toward human tissue kallikrein," The Journal of Biological Chemistry, vol. 275, no. 49, pp. 38457-38466, 2000.

[8] R. Q. Miao, V. Chen, L. Chao, and J. Chao, "Structural elements of kallistatin required for inhibition of angiogenesis," American Journal of Physiology - Cell Physiology, vol. 284, no. 6, pp. C1604-C1613, 2003.

[9] H. Yin, L. Gao, B. Shen, L. Chao, and J. Chao, "Kallistatin inhibits vascular inflammation by antagonizing tumor necrosis factor- $\alpha$-induced nuclear factor $\kappa \mathrm{B}$ activation," Hypertension, vol. 56, no. 2, pp. 260-267, 2010.

[10] Y. Guo, P. Li, G. Bledsoe, Z. R. Yang, L. Chao, and J. Chao, "Kallistatin inhibits TGF- $\beta$-induced endothelial-mesenchymal transition by differential regulation of microRNA-21 and eNOS expression," Experimental Cell Research, vol. 337, no. 1, pp. 103-110, 2015.

[11] P. Li, Y. Guo, G. Bledsoe, Z. Yang, L. Chao, and J. Chao, "Kallistatin induces breast cancer cell apoptosis and autophagy by modulating Wnt signaling and microRNA synthesis," Experimental Cell Research, vol. 340, no. 2, pp. 305-314, 2016.

[12] J. Chao, J. N. Stallone, Y. M. Liang, L. M. Chen, D. Z. Wang, and L. Chao, "Kallistatin is a potent new vasodilator," The Journal of Clinical Investigation, vol. 100, no. 1, pp. 11-17, 1997.

[13] L. M. Chen, J. Ma, Y. M. Liang, L. Chao, and J. Chao, “Tissue kallikrein-binding protein reduces blood pressure in transgenic mice," The Journal of Biological Chemistry, vol. 271, no. 44, pp. 27590-27594, 1996.

[14] L. M. Chen, L. Chao, and J. Chao, "Beneficial effects of kallikrein-binding protein in transgenic mice during endotoxic shock," Life Sciences, vol. 60, no. 17, pp. 1431-1435, 1997.

[15] J. Chao and L. Chao, "Biochemistry, regulation and potential function of kallistatin," Biological Chemistry Hoppe-Seyler, vol. 376 , no. 12 , pp. 705-713, 1995.

[16] L. M. Chen, Q. Song, L. Chao, and J. Chao, "Cellular localization of tissue kallikrein and kallistatin mRNAs in human kidney," Kidney International, vol. 48, no. 3, pp. 690-697, 1995.

[17] W. C. Wolf, R. A. Harley, D. Sluce, L. Chao, and J. Chao, "Localization and expression of tissue kallikrein and kallistatin in human blood vessels," Journal of Histochemistry \& Cytochemistry, vol. 47, no. 2, pp. 221-228, 1999.

[18] J. Chao, A. Schmaier, L. M. Chen, Z. Yang, and L. Chao, "Kallistatin, a novel human tissue kallikrein inhibitor: levels in body fluids, blood cells, and tissues in health and disease," The Journal of Laboratory and Clinical Medicine, vol. 127, no. 6, pp. 612-620, 1996.

[19] J. Chao, G. Bledsoe, and L. Chao, "Protective role of kallistatin in vascular and organ injury," Hypertension, vol. 68, no. 3, pp. 533-541, 2016.

[20] R. Q. Miao, J. Agata, L. Chao, and J. Chao, "Kallistatin is a new inhibitor of angiogenesis and tumor growth," Blood, vol. 100, no. 9, pp. 3245-3252, 2002.

[21] B. Shen, M. Hagiwara, Y. Y. Yao, L. Chao, and J. Chao, "Salutary effect of kallistatin in salt-induced renal injury, inflammation, and fibrosis via antioxidative stress," Hypertension, vol. 51, no. 5, pp. 1358-1365, 2008. 
[22] P. Li, G. Bledsoe, Z. R. Yang, H. Fan, L. Chao, and J. Chao, "Human kallistatin administration reduces organ injury and improves survival in a mouse model of polymicrobial sepsis," Immunology, vol. 142, no. 2, pp. 216-226, 2014.

[23] P. Li, Y. Guo, G. Bledsoe et al., "Kallistatin treatment attenuates lethality and organ injury in mouse models of established sepsis," Critical Care, vol. 19, no. 1, p. 200, 2015.

[24] L. Lu, Z. Yang, B. Zhu et al., "Kallikrein-binding protein suppresses growth of hepatocellular carcinoma by anti-angiogenic activity," Cancer Letters, vol. 257, no. 1, pp. 97-106, 2007.

[25] B. Shen, L. Gao, Y. T. Hsu et al., "Kallistatin attenuates endothelial apoptosis through inhibition of oxidative stress and activation of Akt-eNOS signaling," American Journal of Physiology - Heart and Circulatory Physiology, vol. 299, no. 5, pp. H1419-H1427, 2010.

[26] J. Chao, L. M. Chen, K. X. Chai, and L. Chao, "Expression of kallikrein-binding protein and alpha 1-antitrypsin genes in response to sex hormones, growth, inflammation and hypertension," Agents and Actions Supplements, vol. 38, Part 1, pp. 174-181, 1992.

[27] B. Shen, L. Chao, and J. Chao, "Pivotal role of JNK-dependent FOXO1 activation in downregulation of kallistatin expression by oxidative stress," American Journal of Physiology - Heart and Circulatory Physiology, vol. 298, no. 3, pp. H1048H1054, 2010.

[28] J. Chao, P. Li, and L. Chao, "Kallistatin suppresses cancer development by multi-factorial actions," Critical Reviews in Oncology/Hematology, vol. 113, pp. 71-78, 2017.

[29] Y. Guo, P. Li, L. Gao et al., "Kallistatin reduces vascular senescence and aging by regulating microRNA-34a-SIRT1 pathway," Aging Cell, vol. 16, no. 4, pp. 837-846, 2017.

[30] S. L. Lu, C. Y. Tsai, Y. H. Luo et al., "Kallistatin modulates immune cells and confers anti-inflammatory response to protect mice from group A streptococcal infection," Antimicrobial Agents and Chemotherapy, vol. 57, no. 11, pp. 5366-5372, 2013.

[31] J. Chao and L. Chao, "A major difference of kallikrein-binding protein in spontaneously hypertensive versus normotensive rats," Journal of Hypertension, vol. 6, no. 7, pp. 551-558, 1988.

[32] C. Chao, P. Madeddu, C. Wang, Y. Liang, L. Chao, and J. Chao, "Differential regulation of kallikrein, kininogen, and kallikrein-binding protein in arterial hypertensive rats," American Journal of Physiology - Renal Physiology, vol. 271, no. 1, pp. F78-F86, 1996.

[33] N. D. Vaziri, "Causal link between oxidative stress, inflammation, and hypertension," Iranian Journal of Kidney Diseases, vol. 2, no. 1, pp. 1-10, 2008.

[34] A. Modaresi, M. Nafar, and Z. Sahraei, "Oxidative stress in chronic kidney disease," Iranian Journal of Kidney Diseases, vol. 9, no. 3, pp. 165-179, 2015.

[35] F. J. Pashkow, "Oxidative stress and inflammation in heart disease: do antioxidants have a role in treatment and/or prevention?," International Journal of Inflammation, vol. 2011, Article ID 514623, 9 pages, 2011.

[36] J. Chao, H. Yin, Y. Y. Yao, B. Shen, R. S. Smith Jr., and L. Chao, "Novel role of kallistatin in protection against myocardial ischemia-reperfusion injury by preventing apoptosis and inflammation," Human Gene Therapy, vol. 17, no. 12, pp. 1201-1213, 2006.

[37] L. Gao, H. Yin, R. S. Smith, L. Chao, and J. Chao, "Role of kallistatin in prevention of cardiac remodeling after chronic myocardial infarction," Laboratory Investigation, vol. 88, no. 11, pp. 1157-1166, 2008.

[38] X. Huang, X. Wang, Y. Lv, L. Xu, J. Lin, and Y. Diao, "Protection effect of kallistatin on carbon tetrachloride-induced liver fibrosis in rats via antioxidative stress," PLoS One, vol. 9, no. 2, article e88498, 2014.

[39] Y. Liu, G. Bledsoe, M. Hagiwara, B. Shen, L. Chao, and J. Chao, "Depletion of endogenous kallistatin exacerbates renal and cardiovascular oxidative stress, inflammation, and organ remodeling," American Journal of Physiology - Renal Physiology, vol. 303, no. 8, pp. F1230-F1238, 2012.

[40] L. Gao, P. Li, J. Zhang et al., "Novel role of kallistatin in vascular repair by promoting mobility, viability, and function of endothelial progenitor cells," Journal of the American Heart Association, vol. 3, no. 5, article e001194, 2014.

[41] C. Zhu, F. Pan, L. Ge et al., "SERPINA3K plays antioxidant roles in cultured pterygial epithelial cells through regulating ROS system," PLoS One, vol. 9, no. 10, article e108859, 2014.

[42] W. Liu, Q. Chen, J. Liu, and K. Liu, "Normobaric hyperoxia protects the blood brain barrier through inhibiting Nox2 containing NADPH oxidase in ischemic stroke," Medical Gas Research, vol. 1, no. 1, p. 22, 2011.

[43] A. M. Poff, C. Ari, T. N. Seyfried, and D. P. D'Agostino, "The ketogenic diet and hyperbaric oxygen therapy prolong survival in mice with systemic metastatic cancer," PLoS One, vol. 8, no. 6, article e65522, 2013.

[44] P. Rodriguez-Miguelez, E. Lima-Cabello, S. Martinez-Florez, M. Almar, M. J. Cuevas, and J. Gonzalez-Gallego, "Hypoxiainducible factor-1 modulates the expression of vascular endothelial growth factor and endothelial nitric oxide synthase induced by eccentric exercise," Journal of Applied Physiology, vol. 118, no. 8, pp. 1075-1083, 2015.

[45] E. L. Page, G. A. Robitaille, J. Pouyssegur, and D. E. Richard, "Induction of hypoxia-inducible factor- $1 \alpha$ by transcriptional and translational mechanisms," The Journal of Biological Chemistry, vol. 277, no. 50, pp. 48403-48409, 2002.

[46] F. Alavian, S. Hajizadeh, M. R. Bigdeli, and M. Javan, "The role of protein kinase $\mathrm{C}$ in ischemic tolerance induced by hyperoxia in rats with stroke," EXCLI Journal, vol. 11, pp. 188-197, 2012.

[47] S. Zara, V. Macchi, R. De Caro, M. Rapino, A. Cataldi, and A. Porzionato, "pPKC $\alpha$ mediated-HIF- $1 \alpha$ activation related to the morphological modifications occurring in neonatal myocardial tissue in response to severe and mild hyperoxia," European Journal of Histochemistry, vol. 56, no. 1, p. 2, 2012.

[48] S. Movafagh, S. Crook, and K. Vo, "Regulation of hypoxiainducible factor-1a by reactive oxygen species : new developments in an old debate," Journal of Cellular Biochemistry, vol. 116, no. 5, pp. 696-703, 2015.

[49] M. Borjesson, A. Onerup, S. Lundqvist, and B. Dahlof, "Physical activity and exercise lower blood pressure in individuals with hypertension: narrative review of 27 RCTs," British Journal of Sports Medicine, vol. 50, no. 6, pp. 356-361, 2016.

[50] A. S. Ryan, "Exercise in aging: its important role in mortality, obesity and insulin resistance," Aging Health, vol. 6, no. 5, pp. $551-563,2010$.

[51] R. J. Simpson, T. W. Lowder, G. Spielmann, A. B. Bigley, E. C. LaVoy, and H. Kunz, "Exercise and the aging immune system," Ageing Research Reviews, vol. 11, no. 3, pp. 404-420, 2012. 
[52] T. C. Nordin, A. J. Done, and T. Traustadottir, "Acute exercise increases resistance to oxidative stress in young but not older adults," Age, vol. 36, no. 6, p. 9727, 2014.

[53] N. Sallam and I. Laher, "Exercise modulates oxidative stress and inflammation in aging and cardiovascular diseases," Oxidative Medicine and Cellular Longevity, vol. 2016, Article ID 7239639, 32 pages, 2016.

[54] G. L. Pierce, A. J. Donato, T. J. LaRocca, I. Eskurza, A. E. Silver, and D. R. Seals, "Habitually exercising older men do not demonstrate age-associated vascular endothelial oxidative stress," Aging Cell, vol. 10, no. 6, pp. 1032-1037, 2011.

[55] T. Tanabe, S. Maeda, T. Miyauchi et al., "Exercise training improves ageing-induced decrease in eNOS expression of the aorta," Acta Physiologica Scandinavica, vol. 178, no. 1, pp. 3-10, 2003.

[56] R. Hambrecht, V. Adams, S. Erbs et al., "Regular physical activity improves endothelial function in patients with coronary artery disease by increasing phosphorylation of endothelial nitric oxide synthase," Circulation, vol. 107, no. 25 , pp. $3152-3158,2003$.

[57] J. R. Durrant, D. R. Seals, M. L. Connell et al., "Voluntary wheel running restores endothelial function in conduit arteries of old mice: direct evidence for reduced oxidative stress, increased superoxide dismutase activity and down-regulation of NADPH oxidase," The Journal of Physiology, vol. 587, no. 13, pp. 3271-3285, 2009. 


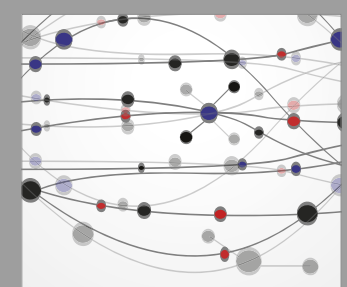

The Scientific World Journal
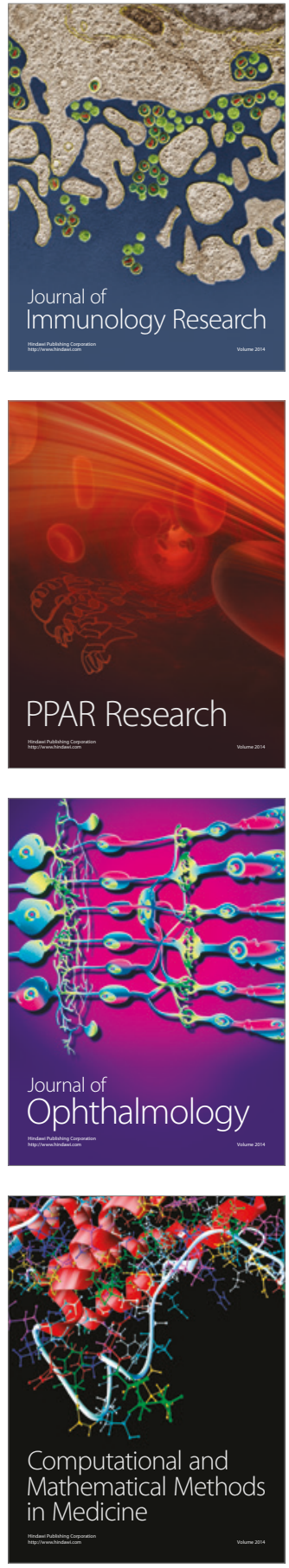

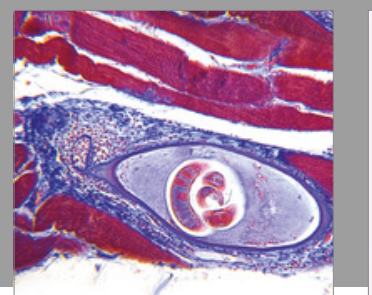

Gastroenterology Research and Practice
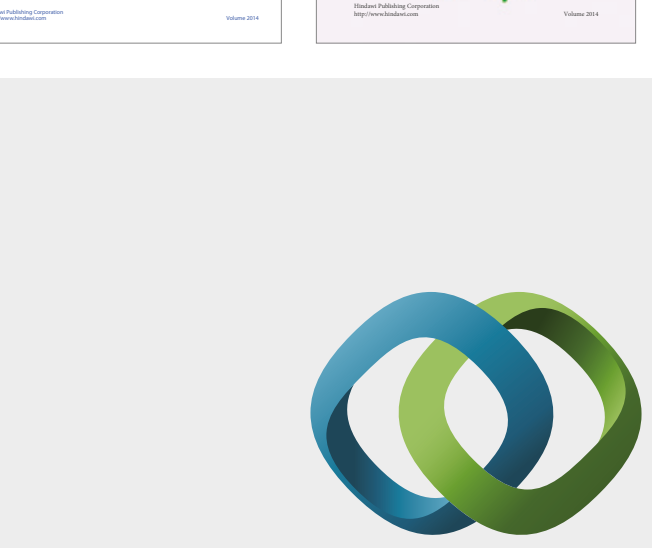

\section{Hindawi}

Submit your manuscripts at

https://www.hindawi.com
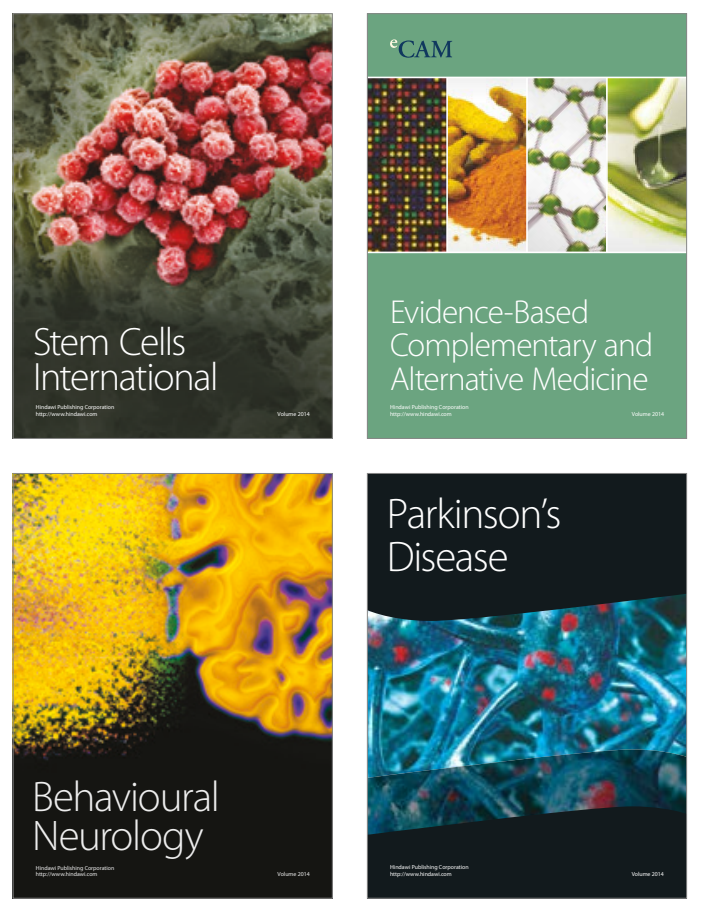
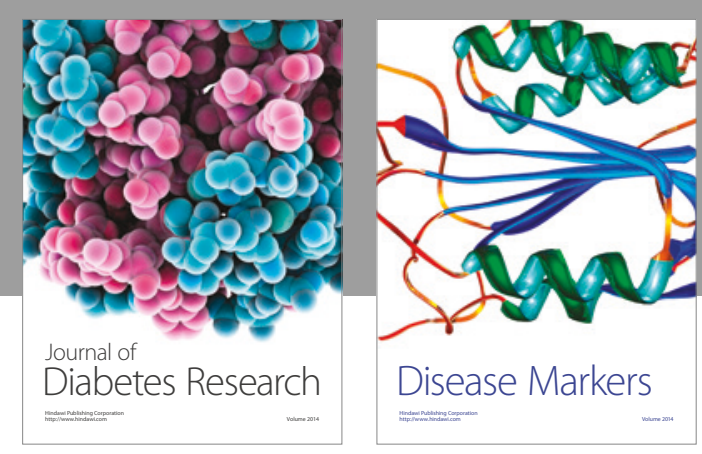

Disease Markers
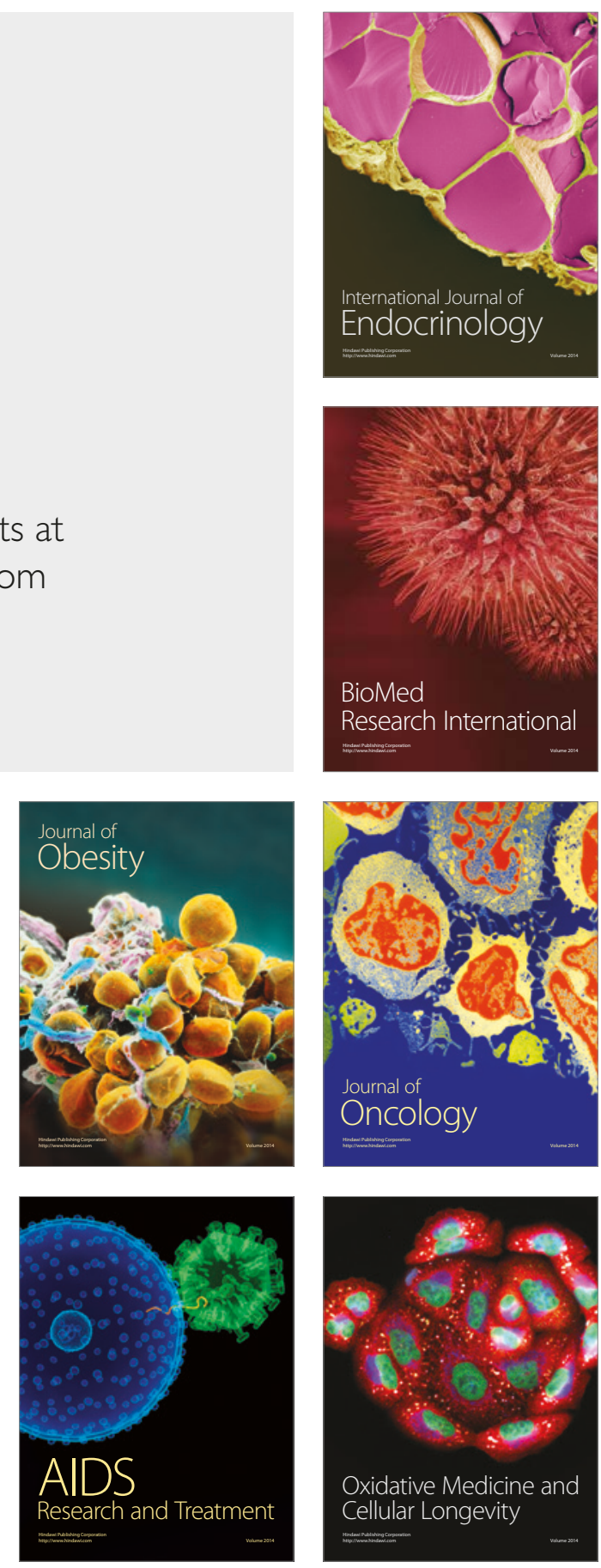